\title{
THE THEORY OF BINAURAL BEATS.
}

By G. W. Stewart.

\begin{abstract}
$A \mathrm{~S}$ described in preceding articles on binaural beats, ${ }^{1}$ there are several phenomena that invite an explanation in terms of physics. The three outstanding aspects are (I) the existence of the "binaural" beats, herein referred to as "primary" beats which correspond to these heard uniaurally with two forks almost in unison, (2) the distinct perception of secondary intensity-maxima when the binaural beat-period is greater than two to five seconds, and (3) the wandering localization of the sound during each beat. We will consider these three separately and propose a theory which seems to promise a satisfactory explanation.
\end{abstract}

Primary Beats.

Dove $^{2}$ in 1839 discovered that when two forks, almost in unison, were presented first to one ear and then, by carrying one about the head, one to each ear, the beats perceived in the first position disappeared and subsequently reappeared as the moving fork approached its final position. I have chosen to call these binaural beats the "primary" beats, for they are always present with observers who are not seriously deaf in one ear. This is not true of the "secondary" beats which occur with clearness only if the primary beat-period exceeds two to five seconds. Separate treatment of the primary beats is justifiable, and in order to make the theory conform strictly to the fact, let us suppose we are dealing with a primary beat-period of approximately one second, or a period where the primary beats alone appear. As already described, ${ }^{3}$ we have then a maximum with $0^{\circ}$ and a minimum with $180^{\circ}$ phase differences.

Let us assume that the forks $F_{1}$ and $F_{2}$ are presented one to each ear, that their amplitudes are equal, that $F_{1}$ has the greater frequency, and that there are conducting paths from each fork to the farther ear.

Let $\epsilon_{1}$ be the retardation in phase in transmission from each fork to the nearer ear. (By the "ear" is meant the physical instrument producing the sensation.)

${ }^{1}$ G. W. Stewart, Phys. Rev., 2d Series, Vol. IX., No. 6, I9I7, p. 502 and p. 509.

${ }^{2}$ H. W. Dove, Repertorium d. Physik, Bd. 3, p. 404, I839.

${ }^{3}$ G. W. Stewart, Phys. Rev., 2d Series, Vol. IX., No. 6, I9ז 7, p. 502. 
Let $\epsilon_{2}$ be the phase retardation in transmission to the farther ear.

Let $\theta$ be the phase of the slower fork. $\theta=2 \pi f t$, where $f$ is the frequency.

Let $\theta+\epsilon$ be the phase of the faster fork.

Let $\alpha$ be the amplitude at the ear produced by the nearer fork.

Let $\beta$ be the amplitude at the ear produced by the farther fork.

The the resulting displacement at the ear nearer to the faster fork, at the instant represented by the value $\theta$, is $\alpha \cos \left(\theta+\epsilon-\epsilon_{1}\right)+\beta \cos \left(\theta-\epsilon_{2}\right)$. At the farther ear the displacement is $\alpha \cos \left(\theta-\epsilon_{1}\right)+\beta \cos \left(\theta+\epsilon-\epsilon_{2}\right)$. By expanding we find the former displacement to be $\sin \theta\left[-\alpha \sin \left(\epsilon-\epsilon_{1}\right)\right.$ $\left.+\beta \sin \epsilon_{2}\right]+\cos \theta\left[\alpha \cos \left(\epsilon-\epsilon_{1}\right)+\beta \cos \epsilon_{2}\right]$ and the maximum displacement, or amplitude, to be

$$
\sqrt{\left[-\alpha \sin \left(\epsilon-\epsilon_{1}\right)+\beta \sin \epsilon_{2}\right]^{2}+\left[\alpha \cos \left(\epsilon-\epsilon_{1}\right)+\beta \cos \epsilon_{2}\right]^{2}} .
$$

The energy of the vibration is proportional to the square of the amplitude. Hence the energy at the ear nearer to the faster fork is,

$$
E_{1}=\alpha^{2}+\beta^{2}+2 \alpha \beta \cos \left(\epsilon-\epsilon_{1}+\epsilon_{2}\right) .
$$

$E_{2}$, or the energy at the ear farther from the faster fork, can be written down by interchanging $\beta$ and $\alpha$ and $\epsilon_{2}$ and $\epsilon_{1}$, or,

$$
E_{2}=\alpha^{2}+\beta^{2}+2 \alpha \beta \cos \left(\epsilon-\epsilon_{2}+\epsilon_{1}\right) .
$$

Let $\epsilon_{2}-\epsilon_{1}$ be represented by $\lambda$.

Then

$$
\begin{aligned}
& E_{1}=\alpha^{2}+\beta^{2}+2 \alpha \beta \cos (\epsilon+\lambda) \\
& E_{2}=\alpha^{2}+\beta^{2}+2 \alpha \beta \cos (\epsilon-\lambda)
\end{aligned}
$$

If the apparent intensity is determined by the sum of $E_{1}$ and $E_{2}$, as we will assume, then,

$$
E_{1}+E_{2}=2\left(\alpha^{2}+\beta^{2}\right)+4 \alpha \beta \cos \epsilon \cos \lambda
$$

and the apparent intensity will be a maximum at $\epsilon=0^{\circ}$ and a minimum at $\epsilon=180^{\circ}$. Moreover, since in the very nature of the case $\beta$ is less than $\alpha$, the minimum can never be a silence. Thus the theory coincides qualitatively with experiment. Quantitative comparison seems impossible.

Discussion.-It will be observed that the above theory does not designate the points of interference in each organ of hearing, but that it does stipulate the occurrence of the interference in a wholly physical instrument. In other words, peripheral interference is accepted as the cause of the primary maxima, or the "beats." To this extent the theory is not new, for Dove, ${ }^{1}$ the discoverer of the primary maxima,

${ }^{1}$ Loc. cit. 
recognized peripheral interference as a possible explanation. This suggestion has often been repeated. Paul Rostosky, ${ }^{1}$ in his theory of the localization and the secondary maxima accepts this non-synchronous variation of the resulting intensity in each ear. But neither in his excellent review ${ }^{2}$ nor in his own contribution just mentioned does Rostosky give a detailed theory explaining the primary maxima and minima.

There may be an objection to the summation of intensities herein employed, but in justification the writer submits four considerations. First, we have the fact that two like tones, each alone faint enough to be just at the limit of hearing, become distinctly audible when simultaneously each is presented to an ear. ${ }^{3}$ Second, summation seems reasonable when one considers the phenomena of the strengthening of intensity in the numerous binaural experiments recorded in Rostosky's review, just mentioned. Third, the experiments of the writer ${ }^{4}$ upon the accuracy of localization of various frequencies, the head not remaining stationary, gives strong evidence in favor of the intensity-sum as an important factor. In these experiments the frequencies most accurately localized are those in which the theoretical variations of the intensity-sum are the greatest. Finally, the perfect blending of the two tones which is actually observed points distinctly to the summation. According to the theory just proposed, the effect is independent of beat-frequency, that is, the primary maxima and minima ought always to appear, which is in accord with fact. The above simple theory would seem to explain the primary maxima and minima satisfactorily. But the phenomena is much more complex, and these additional complexities will be discussed in a following section.

Peterson ${ }^{5}$ discusses the "nature and probable origin" of primary beats and concludes that they are cortical rather than peripheral in origin. His reasons are based upon the evidence of Cross \& Goodwin ${ }^{6}$ to the effect that difference tones cannot be obtained binaurally, the evidence, from experiments on two people each deaf in one ear, that bone conduction must be very small, and upon certain experiments in the counting of the primary beats. The evidence offered by Peterson and the argument in favor of his conclusion are worthy of careful consideration. But in as much as he does not set up any physical theory, a discussion of his paper is probably not appropriate to this article. But the writer should

1 P. Rostosky, Philosophie Studien, I902, I9, p. 557.

2 P. Rostosky, Beiträge Zur Psychologie and Philosophie, I., I905, pp. I73-273.

3 Tarchanow, St. Peterburger Med. Wochensclos., I878, No. 43, (n.R.).

4 G. W. Stewart, Phys. Rev., N.S., Vol. 2, No. r, July, I9r3.

5oseph Peterson, Psychological Review, Vol. XXIII, No. 5, Sept., r9i6.

${ }^{6}$ C. R. Cross \& H. M. Goodwin, Proc. of Academy of Arts \& Science, I89I, 27, I. 
call attention to the fact that the theory of this paper not only assumes a peripheral interference which Peterson denies, but in addition a summation of intensities or a blending which obviously cannot be peripheral.

\section{The Secondary Maxima.}

The only records of the appearance of the secondary maxima are those of Paul Rostosky ${ }^{1}$ and the writer. ${ }^{2}$ Rostosky seeks to explain these maxima which appear first in one ear and then in the other, by a consideration of the localization. He concludes that the ratio of the two intensities, represented by equations (I) and (2), determines the degree of the right or left localizations. If one assumes $\alpha$ and $\beta$ to be of the same order, say I to $\sqrt{2}$ or I to 2 (the values used by Rostosky), and then plots the ratio of $E_{2},(2)$, to $E_{1},(\mathrm{I})$, he will obtain a curve similar to Fig. I. The curve is interpreted by Rostosky as the degree of right localization, the lower half of the curve, inverted, being the degree of left localization. Thus the maximum right localization appears distinctly or extremely right and gives the impression of a maximum intensity. Hence the two secondary maxima occurring in the neighborhood of a difference of phase of $180^{\circ}$ are explained qualitatively; indeed, there also seem to be no objections thereto on the basis of quantitative considerations. Unfortunately, however, Rostosky makes a fatal error in his theory. His equations similar to (I) and (2) are ${ }^{3}$ as follows:

$$
\begin{aligned}
& J_{l}=a^{2}+a_{1}{ }^{2}+2 a a_{1} \cos (x+d / 2) \\
& J_{r}=a^{2}+a_{1}{ }^{2}+2 a a_{1} \cos (x-d / 2) .
\end{aligned}
$$

Where the subscripts $l$ and $r$ refer to the left and right sides respectively, and $a, a_{1}, x$ and $d / 2$ correspond to our $\alpha, \beta, \epsilon$ and $\lambda$, respectively. $d$ is a positive quantity, hence $J_{l}$ reaches a maximum, with changing phase $x$ before $J_{r}$, the former occurring at $x=-d / 2$ and the latter at $x=d / 2$. The faster fork is thus on the left. Moreover, between $x=0^{\circ}$ and $x=\mathrm{I} 80^{\circ}, J_{l}$ is less than $J_{r}$. Experimentally, the secondary maximum appears first (after $x=0^{\circ}$ ) on the side of the faster fork, and hence on the side of the less theoretical intensity. If the maximum in Rostosky's curve, Fig. I, represents localization, then (accepting his equations just given) it must represent "left" localization. But this curve is the ratio $J_{r} / J_{l}$ and therefore must be interpreted as "right" localization. Hence we find that experiment and his theory do not agree. His error is due to a confusion of sides; in fact, one is disposed to guess (incorrectly) that the side of greater intensity (when $0^{\circ}<x<180^{\circ}$ ) is that of the faster fork.

1 Paul Rostosky, Philosophie Studien, I902, I9, p. 557.

${ }^{2}$ G. W. Stewart, Phys. REv., 2d Series, Vol. IX., No. 6, I9I7, 509-5I3.

${ }^{3}$ See loc. cit., p. 580 . 
The writer has considered whether or not an additional assumption of a change in phase during the conduction across the head would lead to a revision of Rostosky's theory that would be in harmony with observation, and he finds a revision upon such an assumption impossible. We are thus led to a complete rejection of the theory of Rostosky.

The writer's theory of the secondary maxima is based upon the outstanding importance of the following experimental facts which have been described in the two preceding articles. ${ }^{1}$

r. The secondary maxima do not occur unless the period of the beats is greater than, say, two to five seconds, whereas the presence of the primary maxima is independent of the beat-period.

2. At the secondary maxima the sound seems localized in the ears (first in the one nearer the faster fork), and between the maxima within the head. With the primary maxima the localization is distinctly external to the head.

3. The sound seems to be much rougher at the secondary than at the primary maxima.

4. The movement of the localization is much more rapid between the two secondary maxima than elsewhere in the rotation.

5. If the secondary maxima appear at phase differences of $180^{\circ}-\delta$ and $\mathrm{I} 80^{\circ}+\delta$ then, for a given fork pitch, $\delta$ is independent of the beatfrequency, but $\delta$ does vary with the frequency of the forks used, and this variation is not a linear one.

6. Unequal exciting intensities at the ears have a tendency to eliminate the secondary maximum on the side of the weaker source.

7. When the beat-frequency is much more rapid than one per second, not only do the secondary maxima disappear but localization in the region of $180^{\circ}$ phase difference also vanishes.

These facts give a definite indication of the difference in origin of the primary and secondary maxima. But before we admit a complexity to account for this difference in origin, let us consider a physically simple equivalent of the skull and ear organs. Let us assume two physical instruments capable of recording vibrations and connected by numerous sound conducting paths. Is it possible for such an arrangement with two beating tones presented simultaneously one to each ear, to make a record that will show the three maxima and three minima actually experienced in each beat-cycle? Let us first assume that each instrument records intensity or a value proportional to the square of the amplitude at the instrument and that the resulting combined record is proportional to the arithmetical sum of these intensities. Upon this assumption we

${ }^{1}$ G. W. Stewart, loc. cit. 
have already found, equation (3), that we may expect only a maximum at $\epsilon=0^{\circ}$ and a minimum at $\epsilon=\mathrm{r} 80^{\circ}$.

If one assumes that the ear is an instrument that records not intensity but the time integral of the absolute value of the displacement, or $\int_{0}^{2 \pi} \mid$ displacement $\mid d \theta,{ }^{1}$ he finds that the record in the nearer ear is proportional to

$$
\left|\sqrt{\alpha^{2}+\beta^{2}+2 \alpha \beta \cos \left(\epsilon-\epsilon_{1}+\epsilon_{2}\right)}\right|
$$

and in the farther ear to

$$
\left|\sqrt{\alpha^{2}+\beta^{2}+2 \alpha \beta \cos \left(\epsilon+\epsilon_{1}-\epsilon_{2}\right)}\right| .
$$

Reference to the equations just preceding (I) will show that these values are, in fact, the absolute values of the amplitudes. The total record, or the blended sensation, would be proportional to the sum of these two absolute values. It can be shown that if $\cos \left(\epsilon_{2}-\epsilon_{1}\right)$ is greater than $2 \alpha \beta /\left(\alpha^{2}+\beta^{2}\right)$ this sum will be a maximum at $0^{\circ}$ and a minimum at $180^{\circ}$. Obviously, $\cos \left(\epsilon_{2}-\epsilon_{1}\right)$ is nearly unity, whereas the term $2 \alpha \beta /\left(\alpha^{2}+\beta^{2}\right)$ is, in all likelihood, much less than unity. We conclude that the foregoing assumption as to the dependence of the sensation upon the absolute value of the amplitude at the physical instrument leads to the same maximum and minimum as that based upon the dependence of the sensation upon the intensity of vibration. This leads one to doubt whether any assumption can be made as to the record of the instrument, the ear, which will result in a total sensation that has three maxima and three minima in one beat-cycle. At any rate, lacking such an assumption, the conclusion is that we must consider the ear not a single instrument, even for one tone. If, for our present purpose, one can consider each ear to consist of two different physical instruments which act somewhat independently of one another, a distinct gain is made thereby. For, assuming the sensations to be non-interfering, it is obvious that the sensations possible with two somewhat independent instruments are very different than those possible with a single instrument. For, suppose that instrument number one vibrates with the resulting vibration $f(t)$ giving the appropriate sensation, and that instrument number two vibrates with the resulting vibration $F(t)$, giving its appropriate sensation. If the sensations are non-interfering, the total sensation cannot, in general, be the same as had the vibrations $f(t)$ and $F(t)$ been combined in a single instrument.

Our theory will assume the equivalent of two physical instruments

1 The writer is indebted for the suggestion of this assumption to Professor R. P. Baker, of the Department of Mathematics in the State University of Iowa. 
in each ear excited in a mechanically different manner. A pair of like instruments, one on each side, is responsible for the primary maxima and minima, and another pair for the secondary maxima and minima. The former pair is excited chiefly through the ear drum-skin. The latter is excited chiefly through the vibrations of the skull. We will assume the mechanical arrangement to be such that the vibration conveyed by the skull from one fork to the distant ear has its phase determined not only by the equivalent length of path, but also by a change in phase of $180^{\circ}$. We will first discuss the consequence of the foregoing assumptions, and then its agreement with experiments.

The pair of A instruments, assumed excited chiefly through the membrana tympani, will give the primary maxima and minima provided we can also assume a conduction from one ear to the other. This conduction can occur by many paths and has been demonstrated actually to exist, ${ }^{1}$ but the methods used could not exhibit the excellence of the conduction. We will assume this conduction as a fact without attempting to describe it in detail. Thus our previous theory, equation (3), can be regarded as applicable to the $\mathrm{A}$ instruments, and the primary maxima are completely explained.

The intensities in the B instruments can best be understood by reverting to the equations from which (I) and (2) were obtained. We will use $\alpha_{1}, \beta_{1}, E_{1}{ }^{\prime}, E_{2}{ }^{\prime}, \epsilon_{2}{ }^{\prime}$ and $\epsilon_{1}{ }^{\prime}$ instead of $\alpha, \beta, E_{1}, E_{2}, \epsilon_{2}$ and $\epsilon_{1}$ respectively, inasmuch as the values for the $\mathrm{B}$ instruments are different. Remembering the assumption that $\epsilon_{2}$ really becomes $\epsilon_{2}{ }^{\prime}+180^{\circ}$ we obtain the displacement at the nearer ear, $\alpha_{1} \cos \left(\theta+\epsilon-\epsilon_{1}{ }^{\prime}\right)+\beta_{1} \cos \left(\theta-\epsilon_{2}{ }^{\prime}-180^{\circ}\right)$ and at the farther ear, $\alpha_{1} \cos \left(\theta-\epsilon_{1}{ }^{\prime}\right)+\beta_{1} \cos \left(\theta+\epsilon-\epsilon_{2}{ }^{\prime}-180^{\circ}\right)$. The intensities at the nearer and farther ears become, substituting $\lambda^{\prime}$ for $\epsilon_{2}{ }^{\prime}-\epsilon_{1}^{\prime}$,

$$
\begin{aligned}
& E_{1}{ }^{\prime}=\alpha_{1}{ }^{2}+\beta_{1}{ }^{2}-2 \alpha_{1} \beta_{1} \cos \left(\epsilon+\lambda^{\prime}\right) \\
& E_{2}{ }^{\prime}=\alpha_{1}{ }^{2}+\beta_{1}{ }^{2}-2 \alpha_{1} \beta_{1} \cos \left(\epsilon-\lambda^{\prime}\right) .
\end{aligned}
$$

Taking the sum of intensities as before, we have,

$$
E_{1}^{2}+E_{2}^{2}=2\left(\alpha_{1}^{2}+\beta_{1}^{2}\right)-4 \alpha_{1} \beta_{1} \cos \epsilon \cos \lambda^{\prime}
$$

This sum has a maximum at $180^{\circ}$ and a minimum at $0^{\circ}$. If the total sensation varies as the sum of equations (3) and (6), or the total intensity sum of both pairs of instruments $A$ and $B$, then, no matter what values are assigned to our constants, we could obtain only one maximum and one minimum for one beat-cycle. This can be shown by the usual method of determining maxima and minima. But the assumption that

${ }^{1}$ K. L. Schaefer, Pflüg. Arch., Bd. 6I, p. 544, I895, and also C. S. Myers and H. A. Wilson, Proc. Roy. Soc., I908, A 80, p. 260. 
insturments $\mathrm{A}$ and $\mathrm{B}$ are differently excited, leads us to assume also a difference in sensation from these excitations. If $\alpha_{1}$ and $\beta_{1}$ have nearly the same value the intensity sum of the $B$ instruments will be a maximum at $180^{\circ}$ and a minimum, almost zero, at $0^{\circ}$ phase differences. We would expect, then, the resulting observed effects at $0^{\circ}$ to be chiefly the intensity-sum produced by the $\mathrm{A}$ instrument and at $\mathrm{I} 80^{\circ}$ to be the combination of the minimum of the A-intensity-sum and the somewhat different maximum of the B-intensity-sum. This is precisely the impression that an observer first obtains. He does not find two secondary maxima but only one in the neighborhood of $180^{\circ}$ phase difference. Further, this maximum at $180^{\circ}$ as already stated, appears to come from a source located in or on the skull, whereas the maximum at $0^{\circ}$ is externally localized. This effect is in entire agreement with the assumption that the $B$ instruments are excited by a skull vibration and the $A$ instruments by an aërial vibration in the external meatus. The fact that the $180^{\circ}$ maximum does not appear unless the beat-period is longer than two to five seconds indicates that the excitation of the $B$ instruments requires a longer time, or that the inertia of the system is large, and this is in harmony with the assumption just mentioned.

But how account for two secondary maxima? In discussing the addition of intensities with the $\mathrm{A}$ instruments, we tacitly assumed a blending of the sound so that the maximum in either instrument was not noticed. This blending is, in fact, a part of our usual experience. But we have no reason for believing that the usual degree of blending must occur with the intensities at the $\mathrm{B}$ instruments. Indeed, the cause of blending (with the A instruments) is not understood. We may, therefore, without making any additional assumptions investigate what the effect would be if the observer is able to recognize the maximum intensity in each B instrument. Equations (4) and (5) show that the maximum intensity in the $\mathrm{B}$ instrument located on the side nearer the faster fork occurs before the maximum in the other B instrument by a phase of $2 \lambda^{\prime}$. Therefore, the maximum at the nearer $B$ instrument would occur at $180^{\circ}-\lambda^{\prime}$ and at the farther $B$ instrument at $180^{\circ}+\lambda^{\prime}$ phase differences. Further, the localization, because the excitation is from the skull, would appear to be first in the nearer and then in the farther ear. Again the theory is in distinct harmony with experiment. Thus, by assuming a difference between the $\mathrm{A}$ and $\mathrm{B}$ instruments in the manner of excitation and a difference in the degree of blending of the sensations, we can account for the appearance of the secondary maxima.

The difference in localization in the two parts of the beat-cycle, near $0^{\circ}$ and near $180^{\circ}$ phase difference, will be discussed in detail on a succeed- 
ing page, but the comment may here be made that the cause is to be found in the difference in the manner of excitation of the two pairs of instruments.

The difference in the roughness of the sound in the two parts of the beat-cycle may also be explained by differences in excitation, for the excitation via the membrana tympani does, as we know, enable us to detect purity of tone, whereas the excitation directly by the skull would undoubtedly include natural vibrations of the skull itself and thus produce a somewhat rougher sound.

The rapid movement of the localization will also be presently discussed and satisfactorily explained on the basis of our theory.

The fact that $\delta$ (or $\lambda^{\prime}$ in our theory) is independent of the beat-frequency meets ready explanation for $\lambda^{\prime}$ represents the difference in retardation due to difference in the paths to the nearer and farther $B$ ear, and certainly this value depends only upon the frequency of vibration and the conducting paths and not upon the rate at which the phase differences of the sources are changing. Or, in other words, $\lambda^{\prime}$ is entirely independent of $\epsilon$ and $d \epsilon / d t$.

But how does theory agree with the variation in $2 \lambda^{\prime}$ (our earlier $2 \delta$ ) with frequency, as shown in Fig. I of a previous article?' ${ }^{1}$ We have assumed a retardation in phase of $\epsilon_{1}^{\prime}$ in the propagation from the source

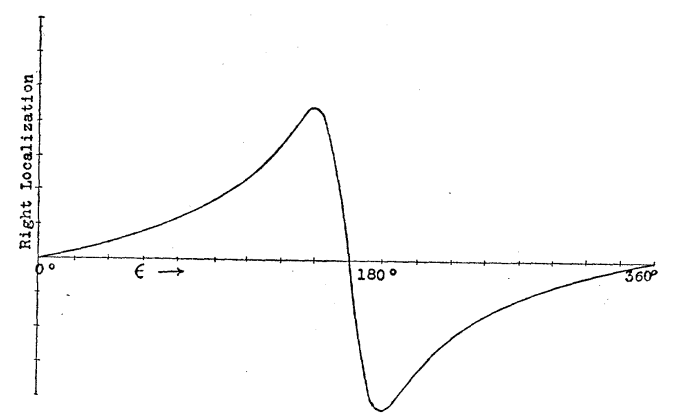

Fig. 1.

to the nearer instrument, and a retardation of $\epsilon_{2}^{\prime}$ in the path from the source to the farther instrument. The excess retardation in conduction to the farther ear is thus $\epsilon_{2}^{\prime}-\epsilon_{1}^{\prime}$ or $\lambda^{\prime}$. We can now introduce a helpful simplification without sensible error. Assume $\epsilon_{1}^{\prime}$ occurs in a single path. Then it will be proportional to the frequency. But $\epsilon_{2}{ }^{\prime}$ will be a resultant of conduction over an indefinite number of paths, with varying amplitudes. Can $\epsilon_{2}^{\prime}$ be regarded as occurring in an equivalent single path

${ }^{1}$ G. W. Stewart, Phys. Rev., 2d Series, Vol. IX., No. 6, 1917, pp. 507-513. 
and hence be considered as proportional to frequency? As we will see, the answer is a negative one. For let us consider two paths only, along which the vibrations have the amplitudes $a_{1}$ and $a_{2}$. The difference in time required to transverse these paths is constant and hence the difference in phase at the distant junction of the paths is proportional to the frequency. Let this phase difference be $\gamma$, let $r$ be the resultant amplitude, and let $\epsilon^{\prime}$ be the phase difference between the resultant vibration and the one proceeding along the longer path, say, $a_{2}$.

Then

and

$$
r^{2}=a_{1}^{2}+a_{2}^{2}+2 a_{1} a_{2} \cos \gamma
$$

$$
r \sin \epsilon^{\prime}=a_{1} \sin \gamma
$$

From these equations we get, in case $a_{1}=a_{2}$,

or

$$
2 \cos \frac{1}{2} \gamma \sin \epsilon^{\prime}=\sin \gamma
$$

$$
\epsilon^{\prime}=\gamma / 2 \text {. }
$$

Thus with equal amplitudes, or with $a_{2}=0, \epsilon^{\prime}$ is proportional to $\gamma$ and therefore to the frequency. The assumption of equality of amplitudes 'would therefore give us a change in the phase, $\epsilon_{2}^{\prime}$, that is proportional to the frequency. But the assumption of equality of amplitude is not correct, for different paths would, on account of the differences in length and the presence of damping, have different amplitudes. We are then compelled to reject the suggestion that the various paths can be equivalent to a single path. Our expectation would therefore be that $\lambda^{\prime}$ or $\epsilon_{2}{ }^{\prime}-\epsilon_{1}{ }^{\prime}$ would change either more rapidly or less rapidly than in proportion to the frequency. Our experiments, as already described, show the latter to be the case.

As already stated, if the intensities of the two sources are far from equal, the general effect of a maximum in the neighborhood of $180^{\circ}$ phase difference is retained, but the secondary maximum on the side of the less intensity is very noticeably lessened. This is apparently caused by the fact that there is enough blending of tone to cover up the individual maximum which is, as experiment shows, only recognized at all with practice.

We have thus discussed all the phenomena (excepting those presently to be considered under "localization") and have found that all are distinctly in harmony with the theory herein presented.

\section{The Localization.}

Lord Rayleigh ${ }^{1}$ is inclined to favor perception of phase difference as one of the explanations of the phenomena of localization. At zero-phase

${ }^{1}$ Lord Rayleigh, Proc. Roy. Soc., I909, A 83, pp. 6I-64. 
difference the sound is localized in front from which point it seems to wander about the head in the direction of the side of the tone of higher frequency, then into the ear. It then seems to pass suddenly (at $180^{\circ}$ phase difference) through the head, reappearing in the other ear, and then back to the median plane in front by a path similar to that of the first half of the beat-cycle. C. S. Myers and H. A. Wilson ${ }^{1}$ from experimental evidence showing the variation of the displacement of the localization with changing phase, accept the view that the lateral displacement is proportional to the difference of the intensities at the ears. If we subtract (2) from (I) we get $-4 \alpha \beta \sin \epsilon \sin \lambda$. If now we assume a phase change of $180^{\circ}$ to be introduced in transmission we get, by substituting $\lambda+180^{\circ}$ for $\lambda,+4 \alpha \beta \sin \epsilon \sin \lambda$. The lateral displacement is then proportional to $\sin \epsilon$ and is zero when $\epsilon=0^{\circ}$ or $\mathrm{I} 80^{\circ}$ and is a maximum for $\epsilon=90^{\circ}$. This is the Myers-Wilson theory of localization. It does offer an explanation of the wandering of the sound toward and away from the median plane, but it is objectionable because by using this physical theory one cannot explain the primary maxima, the secondary maxima, the entrance of latter only when the beat-period is sufficiently long, the external localization in the region, $\epsilon=0^{\circ}$, the internal localization in the region $\epsilon=180^{\circ}$, the very small effect of excessive inequalities in intensities upon the localization in the region $\epsilon=0^{\circ}$ and the marked effect in the region $\epsilon=180^{\circ}$, the difference in rapidity of movement in the localization at $\epsilon=0^{\circ}$ and $\epsilon=\mathrm{I} 80^{\circ}$, and the simultaneous disappearance of localization and secondary beats in the region $\epsilon=180^{\circ}$, when the primary beats are much more rapid than one per second..$^{2}$ In fact, the localization in the half cycle $\epsilon=270^{\circ}$ to $2 \pi+90^{\circ}$ differs so much from the localization in the other half that one can hardly accept a theory that does not also make a distinction. In justice to Messrs. Myers and Wilson the statement should be made that their form of apparatus did not lead them to perceive most of the features that have been mentioned.

In the view of the writer the explanation of the localization is not so important as that of the primary and secondary maxima, for while the localization does not occur with all observers even with considerable practice, the presence of the maxima seems common to all. From the physicist's viewpoint, our theory would be that in the phase region, $270^{\circ}<\epsilon<2 \pi+90^{\circ}$, difference of phase at the $\mathrm{A}$ instruments ${ }^{3}$ is a

${ }^{1}$ C. S. Myers and H. A. Wilson, Proc. Roy. Soc., I908, A 80, p. 260.

2 Results given by G. W. Stewart, Phys. Rev., 2d Series, Vol. IX., No. 6, I91 7, pp. 502-508.

${ }^{3}$ For our present purpose it maybe sufficient to note that the phase of the fork nearer to an ear will furnish the greater portion of the amplitude and thus that the variation in phase difference at the $\mathrm{A}$ instruments is largely determined by the phase difference of the forks themselves. 
determining factor, and that in the region $90^{\circ}<\epsilon<270^{\circ}$ the nonblending excitation of the $\mathrm{B}$ instruments, first with greater intensity on the side of the higher pitch, and then with equal intensity and then with greater intensity on the other side, explains the localization. The explanation of the region $270^{\circ}<\epsilon<2 \pi+90^{\circ}$ by phase difference will not meet the approval of many psychologists, on the ground that sensation depends upon the mode of response of the end organ and not upon the character of the stimulus. But one must not treat too seriously the application of a generalization that has never been definitely established. Moreover, inasmuch as all observers do not obtain the variation of localization herein described, we may assume it to be a "second-order effect" or one whose cause may never have been detected in experiments with sensory stimuli. Peterson ${ }^{1}$ considers the localization in this region as caused by phase differences and concludes that the perception of phase difference is cortical in origin. The explanation given above of the localization in the region $90^{\circ}<\epsilon<270^{\circ}$ does not meet with the objection just cited or any other of which the author is aware. Moreover, it should be anticipated that the excitation of the A instruments by the usual route, membrana tympani, etc., would give an external localization and the excitation of the $\mathrm{B}$ instruments, directly by the skull, an internal localization, just as is always produced by any vibration given the skull only. The effects produced by unequal intensities at the ears also accord with the theory. For if the localization near $\epsilon=0^{\circ}$ is due chiefly to a perception of phase difference by the A instruments the effect of unequal intensity should be slight. Again, if the localization near $\epsilon=180^{\circ}$ is caused by the lack of complete blending of the intensities at the $\mathrm{B}$ instruments, it would be seriously modified by inequality in the intensities at the ears. Both of these conclusions are verified by experiment. The rapid movement of the localization in the region of $180^{\circ}$ is in accord with the theory, which gives the variation in phase difference with localization in the ears as $2 \lambda^{\prime}$ or $2\left(\epsilon_{2}^{\prime}-\epsilon_{1}^{\prime}\right)$, for, from physical considerations, we can see that $\epsilon_{2}{ }^{\prime}-\epsilon_{1}{ }^{\prime}$ must be a small angle. Suppose the equivalent difference of path in the skull bones were $20 \mathrm{~cm}$. If this were in air, the phase introduced thereby for a frequency of 256 d.v. would be about $60^{\circ}$. But the value $\lambda^{\prime}$ or $\delta$ for this frequency is approximately $45^{\circ} .^{2}$ Inasmuch as the velocity in the skull bones must be several times ${ }^{3}$ that in the air, we find that the theory would suggest an angle even smaller than that found by experiment. Both theory and experiment agree qualitatively that the dif-

${ }^{1} \mathrm{~J}$. Peterson, loc. cit.

${ }^{2}$ G. W. Stewart, PHys. Rev.

${ }^{8}$ H. Frey, Ztschr. f. Psych. u. Phys. d. Sinnesorgane, Bd. 28, p. 9, I902, gives ten times. 
ference between the phase relations at the two secondary maxima is markedly less than $180^{\circ}$ and hence that the passage from the first secondary maximum (following $0^{\circ}$ phase difference) to the other would seem to be more rapid than the movement in the remainder of the beat-cycle. The quantitative agreement is however, not satisfactory.

The theory is further confirmed by the fact that with a rapid beatfrequency (more rapid than one per second) not only do the secondary maxima disappear, but the localization in the $180^{\circ}$-phase-difference region disappears also.

\section{Anatomical Considerations.}

The query arises, does the structure of the ear permit of the assumptions as made? It would seem that no serious objection arises if we assume the instrument $A$ to be the organs of Corti in the ductus cochlearis, and the instrument $B$ to be the similar organs in the saccule or the utricle. These organs in the cochlea and the saccule and utricle, have a common origin. Herrick ${ }^{1}$ states that some physiologists have thought that cochlear and vestibular nerve systems are not wholly distinct and that the sense organs in the saccule may also function as a sound perceptor. This uncertainty concerning the function of the organs in the utricle and saccule on the part of the physiologists does not serve as an objection to our theory. Indeed, the fact that the complex phenomena herein described can be accounted for by the assumption of the existence of the B instruments ought to assist in deciding this uncertainty. The fact that there is a great difference in the central connections of the cochlear and vestibular nerves is in harmony with our contention that the instruments $\mathrm{B}$ give sensations which do not completely blend and which do not produce external localization.

But are the vestibular and cochlear organs mechanically different? Obviously they are, for the latter are placed upon a membrane, and are apparently excited by the motions of the fluid in the scala media, of the basilar membrane and of the tectorial membrane. The vestibular organs are located at the points where the membranous labyrinth is fastened to the skull itself. Moreover, the movement of the vestibular fluid must be very small for the vestibular canals are closed. Thus there would seem to be a mechanical difference which corresponds admirably with our assumption that instrument $\mathrm{A}$ is excited via the vibration of the membrana tympani and that instrument $B$ is excited by the skull. The only change in our assumption induced by the structure of the ear would

${ }^{1}$ C. J. Herrick, Introduction to Neurology, pp. 20I-202. 
be the possible insertion of the word "chiefly" after each word "excited" occurring in the preceding sentence. ${ }^{1}$

\section{Restatement of Theory and Agreement with Exferiment.}

By the assumption of two physical instruments A and B on each side, having a difference in mechanical arrangement, a difference in the method of excitation, a difference in the blending of the sensations of the A pair and $\mathrm{B}$ pair of instruments and an independence of sensations produced by the two pairs and the additional assumptions that instruments $A$, the usual instruments of hearing, can perceive phase differences, and that in the skull conduction to the $\mathrm{B}$ instruments there is an additional phase change of $180^{\circ}$, we have a theory that explains fairly satisfactorily the presence of the primary maxima, the secondary maxima, the appreciable length of beat-period requisite for the appearance of the latter, the localization in the entire beat-cycle, the difference in the nature of the localization and in the quality of the sound in the two halves of the beatcycle, the rapid movement of localization between the secondary maxima, the variation of the separation of the secondary maxima with the forkfrequency and its independence of beat-frequency, the difference in the effects of unequal intensities upon the localization in the two halves of the beat-cycle, and finally the simultaneous disappearance of the secondary maxima and the localization in the region of $180^{\circ}$-phase-difference.

Our theory does not lead us to identify the B instruments, but merely to suggest the possibility of these instruments being located in the utricule and saccule. Anatomical and physiological considerations give evidence in favor of the suggestions.

The theory is, of course, incomplete, but it has herein proved to be very successful and hence promising. There are no conspicuous phenomena connected with binaural beats that are not in accord with the theory, but there are minor points that should receive further consideration. For example, S. P. Thompson ${ }^{2}$ has found binaural beats occurring when the tones used have frequencies of almost two to one.

While it is known ${ }^{3}$ that the secondary maxima and the localization phenomena occur both when the sounds are presented by tubes which

${ }^{1}$ A paper of importance has just come to the writer's notice. D. Richards (Zeitsch. f. Biol., I9I6, 66, 579-609) reports upon experiments with a guinea pig in which there were removed (I) both cochlea, (2) both cochlea and one vestibule and (3) both labyrinths. His conclusion is that sound stimuli may be regarded as adequate stimuli for the vestibular apparatus, but that no conclusion can be drawn as to any sensation produced in this way. This contribution is entirely favorable to the theory presented in this paper.

${ }^{2}$ S. R. Thompson, Phil. Mag., (5), IV, I877, p. 274.

${ }^{3}$ G. W. Stewart, Phys. Rev., 2d Series, Vol. IX. No. 6, I9I7, pp. 502-508. 
approach but do not close the opening of the external meatus, yet there may be a difference in the quantitative measurement of $2 \delta$ with the ear open and closed. The experiments of the writer have been made almost entirely with binaurals that close the external meatus, and accurately speaking his theory has reference to that experimental condition. Nevertheless he believes it to be equally applicable to the open-ear-binaurals.

Physical laboratory,

State University of Iowa. 